\title{
Entre tú y yo. Otra forma de hacer entrevistas: una experiencia radiofónica universitaria
}

institucional.us.es/ambitos/

\author{
María Luisa Sánchez Calero \\ Universidad Complutense de Madrid \\ mlusaca@ucm.es
}

English Version: Between you and me: another way of doing interviews, auniversity radio experience

\begin{abstract}
Resumen
Los avances tecnológicos aparecidos en el campo de la información y la comunicación, propician la aparición de plataformas audiovisuales que con la adaptación del sistema universitario español al Espacio Europeo de Educación Superior (EEES), favorecen la incorporación de nuevas metodologías docentes para ajustarse al European Credit Transfer System (ECTS). Una de estas iniciativas es

"Entre tú y yo: otra forma de hacer entrevistas", un programa en Inforadio, la emisora de la Facultad de Ciencias de la Información de la UCM cuyo objetivo es

impulsar la formación teórica y práctica del género de la entrevista dentro del
\end{abstract}

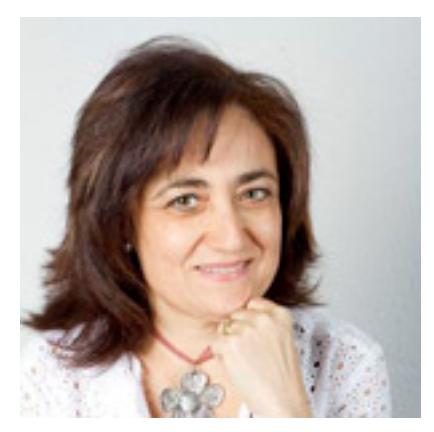

formato radiofónico.

\section{Palabras clave}

Plataformas audiovisuales, entrevista radiofónica, créditos ECTS, tecnología e información.

\section{Abstract}

Technological advances have appeared in the field of information and communication, promoting the emergence of new audiovisual platforms. The adjusting of the Spanish University system to the European Higher Education Area (EHEA) has driven to the incorporation of new teaching methods to fit the university European Credit Transfer System (ECTS). One of the initiatives for student learning in the approach to the EHEA, is "Between you and me: another way of doing interviews," radio programme of the Sciences Information. This channel promotes the education and training on interview's matter in the radio format.

\section{Keywords}

Audiovisual platforms, radio interview, credit transfer system (ECTS), information and technology.

\section{INTRODUCCIÓN}

El avance de las nuevas tecnologías ha cambiado considerablemente el modelo educativo y la radio representa a uno de estos medios tradicionales que bajo las posibilidades de explotación que le brinda Internet incorpora múltiples posibilidades y también múltiples aplicaciones a la docencia. Pero son sus características pedagógicas sumadas a su inmediatez, la fugacidad con la que puede transmitir, la credibilidad de reconocer a sus fuentes de inmediato, los diferentes perfiles de audiencias, o su bajo coste, lo que la convierte en una apuesta segura para cualquier proyecto. Así que estos beneficios unidos a la ya reconocida capacidad pedagógica y formativa del medio ha supuesto la atribución de nuevas competencias dirigidas al ámbito de la enseñanza y, concretamente, en la enseñanza universitaria. Lo que cada día se hace más frecuente observar como las Universidades, tanto públicas como privadas, potencian sus plataformas audiovisuales para incorporar nuevos proyectos y modalidades educativas que faciliten nuevas formas de creación, emisión y difusión de sus contenidos docentes.

El nuevo rol que están alcanzando las radios digitales en la formación universitaria, permite la puesta en marcha de proyectos que aprovechan el potencial de la Red para, desarrollar y enriquecer el aprendizaje con 
programas complementarios que responden a los objetivos formativos del contenido teórico que recibe el alumno a través de sus diferentes asignaturas en la Universidad. Programas que, además, potencian sus capacidades con las utilidades que le brindan sus foros, blogs, chat, etc., y, facilitan una constante interacción entre locutor-emisor-alumno y oyente-público-receptor.

Por todo ello, en este trabajo vamos a referirnos a una iniciativa que llega regulada con la integración del Espacio Europeo de Educación Superior y su mayor transparencia en los procesos de aprendizaje de los alumnos. Un programa que conforma la parrilla de Inforadio, la emisora de la Facultad de Ciencias de la Información: Entre TÚ Y YO. Otra forma de hacer entrevistas. Un proyecto radiofónico que nace como herramienta formativa y complementaria para los alumnos universitarios que cursan la asignatura optativa de Técnica de la Entrevista Periodística, de la licenciatura en Periodismo y al que se incorporan los nuevos alumnos de Grado, pero adaptado a la modalidad de enseñanza del crédito ECTS. Enseñanza donde se prima que el alumno se convierta en responsable activo de la adquisición de sus propios conocimientos.

El uso de los medios de comunicación como recurso docente en las aulas universitarias, en los cursos de primaria, secundaria o en centros juveniles, ha sido y sigue siendo una pauta bastante extendida y habitual en España. Sobre todo en cuanto a la utilización de la radio se refiere, están lo suficientemente demostradas las condiciones que ofrece para convertirse en un medio con gran potencial educativo. Pero también lo es todo aquello que los medios de comunicación publican o emiten ya que se convierte en recurso docente y recurrente por el profesor quien lo utiliza con frecuencia como metodología en el aula. Por tanto, la simbiosis entre los medios de comunicación y el aula queda patente tanto en el uso que se hace de los medios, como del empleo de contenidos periodísticos que siguen una metodología docente.

Este binomio hace que algunos autores como Perona (2009) añadan a la radio la denominación de "radio educativa", entre otras funciones. Incluso habla de tres modalidades en la era digital. La primera corresponde a las cadenas que ofrecen programas educativos. La segunda categoría incluye a las edu-webs radiofónicas que son emisoras que se ocupan de trabajar la radio como uno de los ejes principales para la educación .Y la tercera categoría surgen según el autor, en los centros de enseñanza públicos o privados, sean de primaria, de secundaria o universitarios.

En esta tercera categoría se incluyen a las emisoras con presencia en Internet que cuentan con una parrilla programada con espacios adaptados a cada etapa formativa. Y con una amplia programación en la que tienen cabida todo tipo de géneros periodísticos con una temática variada, entrelazando grandes bloques de información y entretenimiento que invitan a participar a un gran número de alumnos. Y es en esta categoría a la que se refiere Perona, donde se incluye el modelo de emisora universitaria de la Facultad de Ciencias de la Información, que nos ocupa en nuestro artículo. Un modelo para el aprendizaje que abre nuevas vías de desarrollo de las prácticas periodísticas en la nueva cultura docente derivada de la implantación del crédito ECTS.

\section{OBJETIVOS: "EDUTAINMENT”, EN CIENCIAS DE LA INFORMACIÓN}

Como ya se ha comentado en la introducción de este trabajo, este artículo pretende aproximarnos a una de esas experiencias que miran a la radio como uno de los instrumentos principales en la labor formativa y práctica de materias docentes en la Universidad (Oliva, 2006; Pérez Tornero, 2005). Estamos ante un estudio que muestra, analiza y valora el desarrollo que ha tenido uno de los programas pioneros en la parrilla de Inforadio - "Entre TÚ y YO. Otra forma de hacer entrevistas"- y su posterior inclusión, desarrollo y visibilidad alcanzada en el blog http://entretuyyoucm.blogspot.com.es.

Pero antes hablaremos del modelo de emisora en la que está incluido el programa que se describe. Inforadio, responde a una emisora radiofónica creada en el ámbito educativo universitario y que cuenta en la actualidad con una programación de 20 espacios de emisión propia, emitidos de lunes a domingos durante 12 horas diarias de programación en directo y en diferido. Acoge temas y géneros muy diversos en su parrilla donde un $60 \%$ son ocupados por Magazine, $30 \%$ de espacios Informativos y deportivos y un $10 \%$ por el resto de programas. 
El respaldo de la Universidad Complutense y de la propia Facultad de Ciencias de la Información permite alojar a Inforadio como emisora de prácticas para los alumnos en unos espacios habilitados técnicamente para sus emisiones radiofónicas. Una emisora cuyo objetivo es explotar las posibilidades educativas que le brinda un medio como el que nos ocupa y que inicia su actual etapa en el curso 2009-2010, promovida por la implantación del crédito ECTS y la creación del Espacio Europeo de Educación Superior. Es un proyecto formativo y educativo que intenta favorecer el trabajo individual y grupal, de los más de cuatrocientos estudiantes que anualmente pasan para realizar sus prácticas en los diferentes programas de la emisora y con perfiles destinados a alumnos de las tres especialidades que integra la Facultad de Ciencias de la Información: Periodismo, Publicidad y Relaciones Públicas y Comunicación Audiovisual . Y coordinado por un docente responsable del funcionamiento técnico y humano de la emisora nombrado por la Decana de la Facultad.

Y ahora nos centraremos en la descripción y análisis de un proyecto creado en el curso académico 2009-2010 con el objeto de convertirse en un programa de radio para el aprendizaje y puesta en marcha de las prácticas periodísticas en la nueva cultura docente derivada de la implantación del crédito ECTS.

- "Entre TÚ y YO. Otra forma de hacer entrevistas"- constituye un espacio radiofónico creado bajo el marco de la asignatura optativa de la licenciatura en Periodismo; "Técnicas de la Entrevista", que tiene como objetivo docente impulsar la formación teórica y práctica del género de la entrevista dentro del formato radiofónico. Es un programa que incorpora desde sus inicios el concepto de formación del EEES, orientado a aplicar el sistema pedagógico de la materia y proyectarlo en una práctica docente que integre las tres premisas fundamentales del periodismo: Informar, Entretener y Educar.

Práctica que no hubiese sido posible sin la simbiosis entre las prestaciones de un medio clásico como la radio con las posibilidades que incorporan las nuevas tecnologías. Lo que ha permitido que programas como estos existan gracias a la conexión de internet que admite sintonizar la radio a través de un ordenador. O conectarse desde cualquier parte del mundo para participar en un foro de discusión, realizar comentarios en directo, visualizar los rostros de las voces de los locutores, personalizar la audición de programas ya emitidos, «podcastear», etc.

\section{METODOLOGÍA}

El método de trabajo del programa "Entre TÚ y YO. Otra forma de hacer entrevistas", fue diseñado teniendo en cuenta las líneas formativas que marcaba el aprendizaje de la asignatura de "Técnicas de la Entrevista", impartida como materia optativa de segundo ciclo en los estudios de periodismo. El objetivo fundamental del programa fue ampliar la formación práctica en torno a la entrevista en un formato radiofónico. Un proyecto ilusionante e ilusionador que nace como una propuesta de clase y que va consolidándose durante los cuatro años de emisión.

El programa es el resultado de un método de trabajo clásico - convertir los contenidos docentes en contenidos aplicables a una práctica profesional-, para construir un espacio de radio dedicado exclusivamente a la entrevista. La función del profesor de la asignatura y coordinador del programa ha sido la de conducir y orientar al grupo formado por unos veinte alumnos que anualmente constituyen el equipo de entrevistadores. Un programa metodológicamente marcado por las pautas que constituyen la técnica del género de la entrevista: documentación, preparación de los bloques temáticos, ejecución, edición de la entrevista y redacción del perfil del entrevistado, que pone el broche final al diálogo.

La norma del programa siempre ha sido hacer honor a su propio nombre. Y aunque no se inventa otra forma de realizar las entrevistas, si se impone un criterio de trabajo basado en la preparación del género en toda su esencia. Así el objeto de cada entrevista ha sido desde el inicio acercar las curiosidades, vivencias y anécdotas que nos desvela el entrevistado bajo el diálogo de la conversación pública.

Las entrevistas son propuestas por los entrevistadores en la reunión de redacción semanal del equipo donde se presentan, y se planifican las fechas de emisión de las entrevistas. La emisión del programa siempre se realiza en diferido, lo que permite programar cada entrevista realizada y editada en función de la actualidad del tema y del entrevistado. En la ejecución de la entrevista el primer paso se encuentra en la preparación de un 
guión que el alumno diseña atendiendo a los ejes temáticos principales, lo que le proporciona mayor seguridad al enfrentarse a su entrevistado. El guión se elabora en base a la información que se obtiene del entrevistado/a distribuidos en tres o cuatro bloques temáticos que permiten organizar por temas cada apartado de la entrevista y que posteriormente se envía al coordinador del programa para su revisión y aprobación.

Aceptado la organización del guión se ejecuta la entrevista en el lugar pactado con el invitado, que casi siempre acepta la invitación de asistir al estudio de Inforadio lo que proporciona una grabación más profesional. Una conversación en la que se toma como referencia el tiempo de duración del programa, que estará en base a 20 minutos de emisión. Se mantiene un diálogo enfrentándose al interés real que tiene el protagonista o el tema que presenta para reflejarlo en la transcripción de cada una de sus respuestas manteniendo siempre la naturalidad y la cercanía en la conversación con el invitado. Pero la buena conducción del diálogo requiere del interés del alumno-entrevistador en no perder aquellas respuestas que le permitan seguir profundizando en el tema reformulando las denominadas preguntas de estimulación y focalización.

Los aspectos más técnicos los proporciona Inforadio a través de su equipo técnico, quien ayuda en la grabación, realización y control del sonido directamente en sus estudios. Finalizada la entrevista, se escucha atentamente y se prepara su edición atendiendo a los protocolos ya establecidos en el libro de estilo del programa en los que se incluye la música y sintonía del mismo.

Y como colofón a la entrevista se le añade un perfil redactado con pinceladas finas y gruesas, bajo la mirada atenta del personaje entrevistado cuando le observamos y escuchamos a través del lienzo de la entrevista.

Una vez que el trabajo ha quedado finalizado se queda ubicado en la parrilla de Inforadio para emitirse en diferido en nuestro horario habitual de emisión que se marca siempre en función de las necesidades de programación de Inforadio en cada curso académico. Nuestro protocolo establece que la entrevista se emita un día y se repita en otro de la misma semana. Y a partir de aquí cuando se emite la entrevista comienza nuestra difusión por las redes sociales y en el blog del programa dónde una vez que sea incluida se podrá escuchar de forma indefinida.

Y siguiendo el criterio metodológico de evaluación del crédito ECTS, el estudiante de Grado recibe como contraprestación a su trabajo durante un curso académico la asignación de tres créditos optativos mientras que los de licenciatura reciben cinco créditos de libre elección concedido por el Vicerrectorado de Estudios. Una experiencia práctica y complementaria a los contenidos teóricos de la materia que constituye un buen ejemplo de "edutainment", de fusión entre entretenimiento y formación adquirida en la elaboración de cada entrevista ya que la supervisión y corrección permite al alumno la comprobación de sus fallos en cada programa y corregirlos antes de que su trabajo sea definitivamente emitido.

\section{ANÁLISIS Y RESULTADOS}

\section{1. "Entre TÚ y YO. Otra forma de hacer entrevistas" - y su blog http://entretuyyoucm.blogspot.com Docencia de la radio en la clave del EEES}

El espíritu innovador, la posibilidad de formación que representa este programa para los alumnos de periodismo junto al hecho de que el proyecto guarde una estrecha relación con la filosofía ECTS, han sido decisivos para que "Entre Tú y YO" siga emitiéndose.

En el curso académico 2012-2013 el programa ha cumplido cuatro años de emisión en Inforadio superando todas las expectativas previstas. Hoy está consolidado como un proyecto maduro y que empieza a dar sus frutos después de la experiencia adquirida en la retransmisión de más de 100 entrevistas. Trabajo desempeñado con tesón y con la constancia y profesionalidad que siempre ha acompañado al equipo por el que han pasado más de cincuenta alumnos-entrevistadores. Intentando desde su inicio mantener la conversación, con entrevistas que incluyen su propia seña de identidad, bajo la sintonía "Chasing Cars" y con un lema: "que las entrevistas tengan chicha".

Pero la constante innovación del programa ha permitido la apuesta firme y comprometida con las nuevas tecnologías que han facilitado la inclusión de sus perfiles en Facebook y Twitter. Y también la incorporación y 
desarrollo del blog del programa http://entretuyyoucm.blogspot.com en su segundo año donde se pueden escuchar las entrevistas actuales y también las de sus tres etapas anteriores. Un blog que ha permitido escuchar las demandas de aquellos alumnos de la Universidad que realizan su estancia Erasmus en otras universidades europeas, y la de los profesionales de los medios que siguen y animan en las redes sociales y, por supuesto, con los protagonistas; los entrevistados.

La creación del blog del programa se inició en el segundo año de emisión 2010-2011 y fue considerado como un nuevo recurso que aportaría mayor visibilidad a un programa de entrevistas emitido en diferido dentro de la parrilla de Inforadio. El blog en pocas semanas sorprendió con un número elevado de visitas que escuchaban las entrevistas, que realizaban comentarios sobre ellas y que aportaban sus sugerencias para entrevistar a otros personajes de la actualidad informativa del momento. En el mes de octubre de 2012 se llegó a las 5000 visitas y en enero de 2013 se cuadruplicaba su cifra alcanzando un total de 46.063 usuarios. Mientras que en julio de 2013 las visitas no se detienen a pesar de existir periodos de vacaciones en los que normalmente no existen emisiones de nuevas entrevistas y alcanzan las 61.198 visitas.

La metodología de trabajo en cuanto a la distribución y asignación de tareas en el blog se distribuyó en función de las capacidades y preferencias de los alumnos miembros del equipo de entrevistadores. El diseño se realizó en blogspot que permitía un acceso fácil, rápido y gratuito. La página comenzaba por el nombre del programa: Entre Tú y Yo. Otra forma de hacer entrevistas.

En su distribución aparecen nueve pestañas que distribuyen los contenidos del blog: Portada, Equipo 20122013, La historia de Entre Tú y Yo, Contacto, Edición 2012/2013, Ediciones anteriores, Entrevistas transcritas, También puede interesarte y Macking Off. El diseño de la página se divide en tres columnas con varios apartados en cada una de ellas. Cada pestaña de la portada permite el acceso desde ella a la página principal donde su parte central está ocupada con el anuncio de la próxima entrevista presentando con una foto al entrevistado/a y entrevistador/a y el horario previsto de emisión. Más abajo se pueden visualizar y escuchar todavía algunas entrevistas anteriores pinchando sobre ellas. Además de existir otro acceso directo que nos permite leer el perfil o retrato completo del entrevistado.

La parte derecha de la página está ocupada por una columna donde aparecen las redes sociales para que nos puedan seguir en facebook y twitter. Una invitación donde los seguidores del programa pudiesen plantear sus propuestas de entrevistados. Y en otra más abajo aparece alfabéticamente detallado los nombres de todos los entrevistados que han pasado por el programa y donde se pueden escuchar las entrevistas realizadas.

La parte izquierda compuesta por otro de los apartados refleja el horario de emisión de las entrevistas en Inforadio y la programación de las entrevistas que se emitirán durante las próximas semanas. Y más abajo en otro apartado aparecen los entrevistadores que han colaborado en la programación, clasificados por orden alfabético y que permite escuchar las entrevistas realizadas por cada uno de ellos.

\subsection{Balance de resultados en el blog}

En cuanto a los resultados del análisis que arroja el blog se observa un balance muy positivo que permite obtener datos llamativos que evidencian los intereses de los seguidores. Es destacable como en el mes de julio de 2013 las visitas totales registradas son de 61.198, es decir 14.921 visitas más que las obtenidas en febrero de 2013 (46.277 usuarios). Lo que supone un aumento muy significativo respecto al balance realizado en Octubre de 2012 (32.264), por tanto casi 10 veces más que cuando empezó la temporada 2011-2012 (5.000). Además este último mes han ascendido a 2.430 visitas, es decir un ascenso significativo de visitas con respecto al mismo mes de julio del año 2012 puesto que en ese registro se contaron 1.805 visitas.

\subsubsection{Gráfico $n^{\circ} 1$ Ranking de las entrevistas más escuchadas}




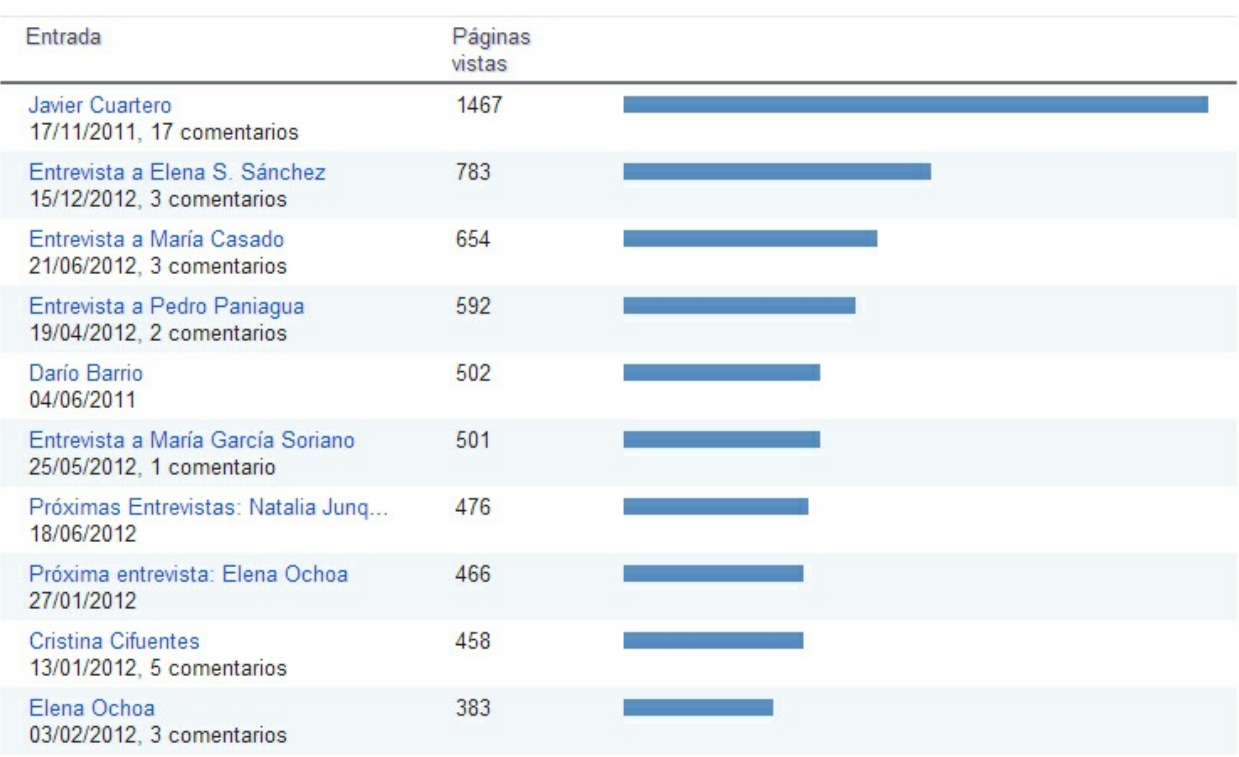

En la imagen se observa que la entrevista a Javier Cuartero, el jugador del equipo Club Balonmano Torrevieja, sigue siendo la líder indiscutible del ranking con 1.467 visitas. Con casi tres veces menos de visitas se sitúa ahora la entrevista a Elena S. Sánchez periodista de los servicios informativos de TVE. Le sigue la entrevista a la periodista María Casado también de TVE. Seguidas por otras como la de Cristina Cifuentes, Delegada del Gobierno en la CAM (anterior entrevista con más visitas en el ranking), el profesor de periodismo Pedro Paniagua, o María García Soriano, antigua compañera del programa. Y seguida en el ranking por el anuncio de entrevistas como la de Natalia Junquera, periodista de EI País. Y en cada una de ellas se reflejan comentarios realizados por los usuarios que han escuchado las entrevistas.

Comentar el puesto en el que se encuentran las entrevistas es un dato revelador que se detecta en la gráfica. Pero esta gráfica muestra los usuarios que han accedido a nuestro blog pinchando directamente en un enlace que le ha llevado hasta la entrevista. Es decir, la entrevista a Javier Cuartero, tiene este número importante de visitas porque el redactor que realizó la entrevista le acompañó de una gran promoción de la misma en periódicos digitales, en las redes sociales y en aquellos foros relacionados con el tema.

Lo mismo ocurre con la entrevista a la Delegada del Gobierno de Madrid, Cristina Cifuentes. El redactor se dedicó a buscar en Google noticias sobre ella y en los comentarios ponía el enlace explicando que era una entrevista muy interesante que le habían realizado los estudiantes de Ciencias de la Información.

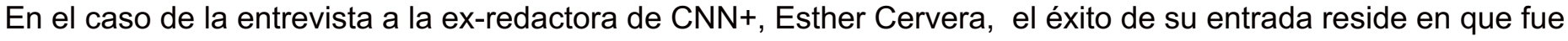
la propia entrevistada la que propagó el link por la Red, empezando por su Twitter y su Facebook.

Este éxito nos llevó a exponer los resultados y proponer en la reunión de redacción que cada redactor se encargase de promocionar en foros o en redes sociales la propia entrevista realizada. Por ejemplo, el día en el que se le realizó la entrevista al Grupo Amaral se entró en la página web del Grupo y se escribió en el foro que se le había realizado una entrevista en el programa. El resultado fue bastante satisfactorio ya que en uno de los comentarios mencionaban al programa y ponían el enlace a nuestro blog.

\subsubsection{Gráfico $n^{\circ}$ 2. Relación de URL de referencia como acceso al programa}


URL de referencia

\begin{tabular}{ll}
\hline Entrada & $\begin{array}{l}\text { Páginas } \\
\text { vistas }\end{array}$ \\
\hline http://www.google.com/search & 1265 \\
https://www.google.es/ & 1023 \\
http://www.facebook.com/ & 333 \\
http://inforadioucm.blogspot.com.es/ & 184 \\
http://inforadioucm.blogspot.com/ & 182 \\
https://www.facebook.com/ & 151 \\
http://balonmano.mforos.com/6617... & 143 \\
http://www.tuenti.com/ & 126 \\
http.//www.entretuyyoucm.blogspot... & 123 \\
http://topblogstories.com/ & 67
\end{tabular}

Este gráfico simboliza como la mayor parte de los visitantes acceden al blog buscando en GOOGLE, es decir, continuando así la tendencia de los últimos tiempos. La diferencia en este año 2013 es que la segunda opción más elegida ha dejado de ser el acceso a través de la web de Inforadio, para ser a través de Facebook. Se comprueba también que otros usuarios acceden desde por tuenti o desde la propia web del programa.

\subsubsection{Gráfico $n^{\circ}$ 3. Relación de palabras claves que simbolizan la entrada de visitantes al programa}

\begin{tabular}{ll} 
Entrada & $\begin{array}{l}\text { Páginas } \\
\text { vistas }\end{array}$ \\
\hline entre tu y yo & 664 \\
maria casado & 339 \\
cristina cifuentes & 291 \\
elena ochoa tve & 123 \\
joseba larrañaga & 75 \\
entre tu y yo ucm & 73 \\
elena s sanchez & 71 \\
dario barrio & 61 \\
maria oña & 61 \\
ana blanco & 59
\end{tabular}

Otra de las cuestiones destacadas en este estudio es la forma de entrada que los visitantes utilizan para acceder al blog del programa. Lo que refleja el gráfico es que emplean una búsqueda directa en GOOGLE colocando las palabras "entre tú y yo" y aparecen aproximadamente 14.200 .000 resultados siendo el blog del programa el primero en aparecer. Por tanto, resulta interesante destacar un nuevo valor añadido a este análisis.

La segunda opción es buscar información localizando directamente el nombre de alguno de nuestros entrevistados (véase por ejemplo Cristina Cifuentes, María Casado, Elena Ochoa o Joseba Larrañaga) y entre los resultados de cada uno aparece un acceso al blog del programa.

Por otro lado, hay que destacar que cada vez son más los usuarios que directamente ponen en la URL el nombre del blog, http://entretuyyoucm.blogspot.com y acceden directamente. Lo que demuestra este dato es que el programa se está creando un hueco entre los mejores programas de entrevistas radiofónicas en España.

\subsubsection{Gráfico $n^{\circ}$ 4. Referencias de las páginas más vistas del blog del programa}

\begin{tabular}{ll} 
Entrada & $\begin{array}{l}\text { Páginas } \\
\text { vistas }\end{array}$ \\
\hline Equipo 2012/2013 & 1465 \\
28/11/2012, 4 comentarios & 684 \\
Ediciones anteriores & \\
$20 / 10 / 2012$ & 643 \\
Making off & \\
$24 / 03 / 2011$ & 633 \\
Contacto & \\
$11 / 06 / 2012$ & 492 \\
También puede interesarte... & \\
21/05/2012 & 362 \\
Entrevistas transcritas & \\
14/01/2013 &
\end{tabular}


En cuanto a las páginas más visitadas al entrar en el blog es la del Equipo que conduce el programa. Lo que demuestra que los usuarios están interesados en conocer quiénes forman el programa y quien lo dirige. Así la renovación del equipo en cada temporada va acompañada de una foto del nuevo grupo y una presentación escrita por la directora del programa donde narra algunos aspectos destacados o novedosos que conformarán la nueva etapa del mismo.

La tercera página más visitada resultó toda una sorpresa porque es la denominada Making Off. Este apartado es uno de los grandes fallos del programa ya que a pesar de la advertencia de la directora por contar anécdotas que marcaron la realización de cada entrevista, no consigue que sus entrevistadores las revelen.

\subsubsection{Gráfico $n^{\circ}$ 5. Procedencia de los usuarios o público del blog del programa}

Por último, resulta obvio, conocer la procedencia de los usuarios o público. Y lo que refleja el gráfico es que la mayor parte procede de España, aunque la sorpresa se encuentra en los seguidores que aparecen en EEUU, México y Perú. Aunque se advierte una mayor presencia de visitas dentro del continente europeo y sobre todo se advierte un aumento significativo desde Francia donde en octubre de 2012 aparecían 609 visitas y en febrero de 2013 la cifra aumentaba hasta las 1719 , un aumento muy significativo. $Y$ en el cuarto puesto del ranking aparece Alemania donde aparecen 765 visitas y se estima que se deben en su gran mayoría a la entrevista realizada al Corresponsal de TVE en Berlín. Respecto al tercer país europeo destacable por sus visitas es Portugal y con un ascenso importante de audiencia durante los últimos meses.

\section{CONCLUSIONES}

En este trabajo se expone la investigación realizada sobre un estudio de caso donde se advierten ciertas tendencias que arrojan un conocimiento útil y provechoso en el ámbito educativo universitario. El estudio relata la experiencia radiofónica de los cuatro

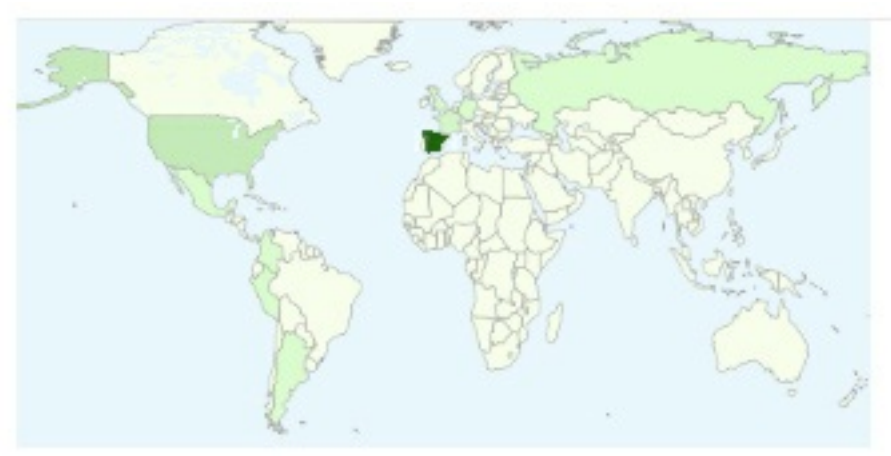

\begin{tabular}{ll} 
Entrada & $\begin{array}{l}\text { Páginas } \\
\text { vistas }\end{array}$ \\
\hline España & 32808 \\
Estados Unidos & 4478 \\
Francia & 1719 \\
Alemania & 765 \\
México & 618 \\
Reino Unido & 572 \\
Colombia & 554 \\
Perú & 460 \\
Rusia & 370 \\
Argentina & 333
\end{tabular}
años de emisión del programa de entrevistas -Entre TÚ y YO: otra forma de hacer entrevistas-. Un programa que se integra en la parrilla de Inforadio, la emisora de Ciencias de la Información en 2010 y que revela datos significativos sobre los beneficios obtenidos a través de la cultura del crédito ECTS en la formación práctica del alumno.

El programa es la validación de un proyecto de periodismo para el aprendizaje en una emisora de radio universitaria incluidas como señala Perona (2009) como radio formativa y cuyo objetivo ha sido contribuir en el aprendizaje de los alumnos de periodismo que deseaban mejorar la técnica del género radiofónico de la entrevista. Los 50 estudiantes que en distintas etapas han colaborado durante estos cuatro años de emisión del programa han valorado positivamente los conocimientos adquiridos, la plataforma, la habilidad y destreza alcanzada en la técnica del género, la planificación y programación así como la utilidad y método de trabajo seguido bajo la supervisión en el proceso de enseñanza y acompañamiento del docente y coordinador del programa.

Este programa y su experiencia han demostrado una vez más que la radio sigue siendo un medio idóneo para el aprendizaje como método docente y sobre todo para las prácticas de enseñanzas periodísticas. Los resultados obtenidos refuerzan dos parámetros que constituyen el periodismo para el aprendizaje: 
entretenimiento y educación. Dos iniciativas que demuestran como los medios de comunicación contribuyen y pueden ubicarse en el centro de cambios sociales que superan los cambios estrictamente informativos. Una perspectiva que según Brown (2002) evoluciona del "infotainment" hacia el "edutainment" (Fossard, 2008) que contribuyen en la transmisión de conocimiento de contenidos educativos con lenguaje y registro y en este caso se suma la de los géneros periodísticos.

Por otra parte, examinadas sus prestaciones es importante destacar la relevancia que en este proyecto ha tenido la creación de su blog http://entretuyyoucm.blogspot.com durante el segundo año del programa. Un blog que ha conseguido que las entrevistas del programa adquieran cada vez mayor visibilidad en muchos ámbitos periodísticos y no periodísticos. Hoy sus entrevistas se están convirtiendo en referentes para los entrevistados y además como fuentes de información para otros compañeros. Pero sobre todo han obtenido por parte del número de usuarios del blog el reconocimiento de calidad que nos garantiza ser fuente fiable en el campo de la información.

El programa ha cumplido cuatro años de emisión en Inforadio superando todas las expectativas previstas por el equipo inicial de integrantes del programa. Hoy está consolidado como un proyecto novedoso y maduro después de la experiencia adquirida en la retransmisión de sus más de 100 entrevistas con entrevistados de reconocimiento y prestigio en su faceta política, la economía, la cultura, ciencia, educación y la comunicación. Entrevistas que son escuchadas por usuarios mayoritariamente procedentes de España, EEUU, México y Perú con casi 5000 visitas de Estados Unidos.

Esta experiencia de programa ha sido el resultado de un método de trabajo clásico -convertir los contenidos docentes en contenidos aplicables a una práctica profesional-, con el objeto de construir un espacio de radio dedicado exclusivamente a la entrevista. La función del profesor de la asignatura y coordinador del programa ha sido la de conducir y orientar al grupo formado por unos veinte alumnos que anualmente formaban el equipo de entrevistadores. Un programa metodológicamente marcado por las pautas que constituyen la técnica del género de la entrevista: documentación, preparación de los bloques temáticos, ejecución, edición de la entrevista y redacción del perfil del entrevistado. Un modelo de trabajo, organización y estilo que han sido el aval para que camine con soltura y confianza haciéndose un hueco en esta nueva era de la información.

Finalmente conviene destacar que estos resultados no pueden entenderse sin un elemento básico que fue que los estudiantes integrantes en el programa tenían que conocer metodológicamente el proceso y técnica de la entrevista lo que supone haber cursado la materia. Este fue el requisito principal del estudio como modelo de programa de refuerzo a la materia cursada y un aprendizaje más práctico y continuado de la misma. Lo que supone una evolución en el "edutainment" con la incorporación de una metodología adecuada que refuerce cualquier nivel de formación práctica a través de la radio.

La experiencia ha resultado positiva, satisfactoria y provechosa para los alumnos y para el docente y puede constituirse como punto de partida de otros proyectos de radio que deben realizarse bajo la cultura del crédito ECTS hoy instalada en los nuevos planes de estudio adaptados a la enseñanza de Bolonia.

\section{APOYOS Y AGRADECIMIENTOS}

Tengo que reconocer el esfuerzo de todos y cada uno de los miembros del equipo del programa -todos aparecen incluidos en el blog - que durante estos cuatro años de emisión han contribuido a la evolución del mismo aportando esfuerzo, dedicación y entusiasmo. Incluso dar las gracias por los momentos menos propicios, cuando en ocasiones recibían alguna negativa a la entrevista propuesta o a los ejes corregidos varias veces o al perfil devuelto con la intención de mejorarlo o incluso el firme propósito de tener que repetir la edición completa de la entrevista.

Pero también agradecer la paciencia y el diálogo de los protagonistas; nuestros entrevistados, que nos han regalado el diálogo de la entrevista y que sin ellos este programa no sería hoy un proyecto hecho realidad.

Para todos y cada uno de ellos, mi más rendido reconocimiento.

\section{REFERENCIAS BIBLIOGRÁFICAS}


CABRERO, J.; LLORENTE, C. \& PUENTES, A. (2009): La satisfacción de los estudiantes en red en la formación semipresencial. Comunicar, nº 35, pp. 149-157. (DOI: 10.3916/C35-2010-03-05)

CODINA, L. (2009): Ciencia 2.0: Redes sociales y aplicaciones en línea para académicos. Hipertext.net, 7.

FLORES, J.M. (2009). Nuevos modelos de comunicación, perfiles y tendencias en las redes sociales.

Comunicar, $\mathrm{n}^{\circ} 33, \mathrm{XVII}, \mathrm{pp} .73-81$.

GARCÍA GALINDO, J.A. (1995): Innovación educativa en la Universidad: investigación y experiencias para mejorar la calidad de la enseñanza. Málaga, Universidad de Málaga.

GIL-DE-ZÚÑIGA, H., JUNG, N. \& VALENZUELA, S. (2012): Social Media Use for News and Individuals Social Capital, Civic Enga -gement and Political Participation. Journal of Computer-Mediated Communication 17, 319336.

GÓMEZ-ESCALONILLA, G, SANTÍN M \&. MATHIEU, G. La educación universitaria on-line en el Periodismo desde la visión del estudiante. Comunicar, n 37 (DOI: 10.3916/C37-2011-02-07) pp.

GUTIÉRREZ, M. \& PERONA, J.J. (2002): Teoría y técnica del lenguaje radiofónico. Barcelona: Bosch.

HANSEN, D.T. (2010): Cosmopolitanism and Education: A View from the Ground. Teachers College Record, 112(1), 1-30.

MARTÍNEZ-RODRIGO, E. \& MARTA-LAZO, C. (2011): Jóvenes interactivos. Nuevos modos de comunicarse. La Coruña: Netbiblo.

PALOMO, B. (2008). Propuesta de un modelo virtual para la asignatura: Periodismo interactivo y Creación de medios digitales. En Análisis, 36; 183-195.

PERONA, J.J. (2009): Edu-webs radiofónicas: experiencias españolas de educación en medios. Comunicar, $n^{\circ}$ 33; pp. 107-114. (DOI: 10.3916/c33-2009-03-001)

PERONA, J.J. \& BARBEITO, M.L. (2007): Modalidades educativas de la radio en la era digital. Icono 14, 9 (www.icono14.net/revista/ núm. 9/ artículos/08.pdf) (24-09-08).

RHEINGOLD, H. (2002): Multitudes inteligentes. La próxima revolución social. Barcelona: Gedisa

SANGRÀ, A. (2008). La integració de les TIC a La universitat: models, problemes i reptes. Tarragona: Universitat Rovira i Virgili.

SANTÍN, M. (2009). La enseñanza on-line en el ámbito de la comunicación en España. Felafacs: XIII Encuentro Latinoamericano de Facultades de Comunicación Social. (www.dialogosfelafacs.net/ -descargas/ APP_EJE2_Espana\%20-\%20Marina\%20Santin.pdf) (14-01-2011).

WILLSON, M. (2010): Technology, Networks and Communities Information, Communication \& Society, 13(5), 747-764. (DOI: 10. 1080/13691180903271572).

\section{Breve semblanza de la autora}

$\mathbf{M}^{\mathbf{a}}$ Luisa Sánchez Calero es doctora en Ciencias de la Información por la Universidad Complutense de Madrid. Actualmente es profesora de la Facultad de Ciencias de la Información y miembro del Grupo de Investigación "MDCS", de la Universidad Complutense, y también del grupo "Riskculture". Sus líneas de investigación se centran principalmente en el estudio del discurso sobre riesgos naturales que emiten los medios de comunicación, cambio climático, meteorología y el estudio de los géneros periodísticos en los medios de comunicación actuales. 
Ámbitos. Revista Internacional de Comunicación, n.27, año 2014, cuarto trimestre (invierno). 